\title{
Clinical features of adenosine sensitive syncope and tilt induced vasovagal syncope
}

\author{
M Brignole, G Gaggioli, C Menozzi, A Del Rosso, S Costa, A Bartoletti, N Bottoni, \\ G Lolli
}

\begin{abstract}
Aim-To evaluate the possible relation between adenosine sensitive syncope and tilt induced vasovagal syncope.

Methods-An ATP test and a head up tilt test were performed in 175 consecutive patients with syncope of uncertain origin. The ATP test consisted of the rapid intravenous injection of $20 \mathrm{mg}$ of ATP; a positive response was defined as the induction of a ventricular pause (maximum RR interval) $\geqslant 6000 \mathrm{~ms}$. The head up tilt test was performed at $60^{\circ}$ for 45 minutes; if negative, 0.4 mg oral glyceryl trinitrate spray was given and the test continued for a further 20 minutes; a positive response was defined as induction of syncope in the presence of bradycardia, hypotension, or both.

Results-Of the 121 patients with a positive response, 77 (64\%) had a positive head up tilt alone, $18(15 \%)$ had a positive ATP test alone, and in $26(21 \%)$ both ATP and head up tilt were positive. Compared with the patients with isolated positive head up tilt, those with isolated positive ATP were older (mean (SD) age, 68 (10) v 45 (20) years), had a lower median number of syncopal episodes $(2 v 3)$, a shorter median duration of syncopal episodes ( $4 v 36$ months), a lower prevalence of situational, vasovagal, or triggering factors $(11 \% v 64 \%)$, a lower prevalence of warning symptoms $(44 \% v 71 \%)$, and a higher prevalence of systemic hypertension $(22 \% v 5 \%)$ and ECG abnormalities ( $28 \% v 9 \%)$. The patients with a positive response to both tests had intermediate features. Of the 44 positive responses to the ATP test, atrioventricular block was the cause of the ventricular pause in 43 ; of the 29 positive cardioinhibitory responses to head up tilt, sinus arrest was present in 23 cases and atrioventricular block in six.

Conclusions-ATP and head up tilt tests identify different populations of patients affected by syncope; these have different general clinical features, different histories of syncopal episodes, and different mechanism sites of action. Therefore, adenosine sensitive syncope and tilt induced vasovagal syncope are two distinct clinical entities. (Heart 2000;83:24-28)
\end{abstract}

Keywords: syncope; adenosine; ATP; head up tilt

Adenosine sensitive syncope has recently been identified as a cause of syncope in some patients affected by unexplained syncope who have an abnormal response to an ATP test and a negative work up after complete conventional investigations. ${ }^{1}$ Some investigators ${ }^{2-4}$ have hypothesised that adenosine could be an important modulator in triggering a vasovagal response in susceptible patients. Indeed, the injection of a bolus of adenosine during head up tilt testing has been seen to provoke a vasovagal response in susceptible patients with syncope, with a positivity rate comparable to that of isoprenaline. ${ }^{23}$ The ATP test has been suggested as a useful tool to identify a subgroup of patients at high risk of severe cardioinhibitory responses of vagal origin. ${ }^{4}$ In the present study we evaluated the possible relation between adenosine sensitive syncope and tilt induced vasovagal syncope. To do this we performed both the ATP and the head up tilt tests in a group of consecutive patients with syncope of uncertain origin and compared the clinical characteristics of the patients who had a positive response to one or both tests.

Correspondence to: Dr Michele Brignole, Via A Grilli 164, 16041 Borzonasca, Italy email: brignole@omninet.it

Accepted for publication 30 July 1999 tigation of syncope between 1 May 1997 and 30 April 1998, in whom the cause of syncope had remained uncertain despite a standardised basic evaluation. This consisted of: a complete history and physical and neurological evaluation; baseline laboratory testing; a 12 lead ECG; ECG monitoring of at least 24 hours' duration; chest $x$ ray examination; $M$ mode and cross sectional echocardiographic evaluation of cardiac function; carotid sinus massage; electrophysiological study (performed in selected patients with structural heart disease or abnormal ECG, or complex premature beats $\left.{ }^{5}\right)$; and further evaluation of any clinical or historical findings suggestive of the cause of the syncope. Patients with historical findings suggestive of vasovagal syncope or situational syncope and negative results in the above

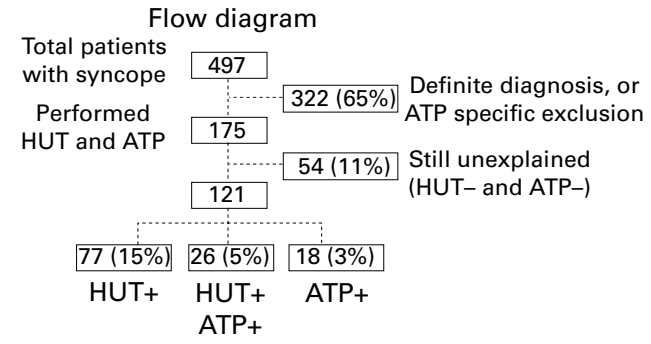

Figure 1 Diagnostic flow diagram of the patients referred for investigation of syncope. HUT, head up tilt test. 
investigations were also included in the study and underwent both head up tilt and ATP testing.

In accordance with published data, ${ }^{6}$ patients with the following characteristics were considered to have a definite or potential cause of syncope and were therefore excluded from the study: positive response to carotid sinus massage according to the "method of symptoms," as previously described ${ }^{7}$; postural hypotension; conversion reaction; seizure disorders; transient ischaemic attack; subclavian steal syndrome; drug induced syncope; aortic stenosis; pulmonary hypertension; hypertrophic cardiomyopathy; arrhythmias (sick sinus syndrome, symptomatic supraventricular tachycardia, second or third degree atrioventricular (AV) block, ventricular tachycardia of more than five beats); and generally accepted abnormalities in the electrophysiological study. ${ }^{58}$ In order to avoid possible confusion in the results of the ATP test, we also excluded 26 patients who had minor electrical abnormalities of impulse formation or of the conduction system (sinus bradycardia $<50$ beats/min, first degree AV block, bundle branch block) or were taking drugs that could impair atrioventricular conduction properties or have potential interactions with ATP (digitalis, $\beta$ blockers, calcium antagonists, antiarrhythmics), or who had already received a pacemaker at the time of syncope.

ATP and head up tilt tests were performed in that sequence during the same day in order to avoid possible daily variations in the clinical conditions, with a time interval between tests sufficient for full recovery of the baseline conditions. The ATP test was performed first as, owing to the very short duration of effect of the drug, it was unlikely to affect the results of head up tilt test.

ATP TEST

ATP (Striadyne, Wieth, France), $20 \mathrm{mg}$, was dissolved in $10 \mathrm{ml}$ of saline solution and injected very rapidly $(<3$ s) into a suitable antecubital vein with the patient in the supine position. No cannulation was used. Continuous recording of the ECG tracing and noninvasive beat to beat arterial blood pressure by the Finapres method ${ }^{910}$ were performed during, and for two minutes after, drug administration. It is well documented ${ }^{24^{11-13}}$ that the maximum bradycardic effect following a bolus of ATP usually occurs after 10-20 seconds (which is the latency time necessary for the drug to reach the heart); this persists for up to 20 seconds and is followed by sinus tachycardia for up to two minutes; hypotension occurs during and immediately after the bradycardic phase and is sometimes followed by moderate hypertension. Facial flushing, shortness of breath, and chest pressure are frequent side effects, but, owing to the rapid deactivation of the drug, these are transient and well tolerated by the patient.

For the purpose of the study, we evaluated the longest RR interval and the maximum drop in systolic blood pressure; this was defined as the difference between the value observed immediately before drug administration and the lowest value observed after it (excluding that of the first two beats following the prolonged asystolic pauses). A positive response to the ATP test was defined as the induction of complete AV block (or sinus pause) with a ventricular pause (maximum RR interval) of $\geqslant 6000 \mathrm{~ms}$, which corresponds to the upper 95th centile of the distribution of values of a control population of subjects without syncope. ${ }^{1}$

HEAD UP TILT TEST

Patients underwent the standardised protocol of upright tilt testing with glyceryl trinitrate challenge currently used in our department for the diagnosis of unexplained syncope..$^{714}$ This consisted of $60^{\circ}$ tilt for 45 minutes or until syncope occurred. If the test did not induce syncope, $0.4 \mathrm{mg}$ oral glyceryl trinitrate spray was given while the patient remained in the same tilting position, and the test was continued for a further 20 minutes. During the test, the beat to beat finger arterial pressure was monitored continuously by the Finapres method. Positive response was defined as induction of syncope in the presence of bradycardia, hypotension, or both. A cardioinhibitory response was defined as the induction of a pause of $\geqslant 3$ s. Mixed or vasodepressor responses were defined when hypotension occurred without a pause of $\geqslant 3$ s. The diagnostic value of upright tilt testing with sublingual glyceryl trinitrate provocation has been validated before, showing a $51 \%$ positivity rate in patients with unexplained syncope and a $6 \%$ false positivity rate in controls. ${ }^{14}$

\section{DEFINITIONS}

A history suggestive of vasovagal syncope was considered to be present if a precipitating event such as fear, severe pain, or instrumentation could be identified. ${ }^{6}$ A history suggestive of situational syncope was considered to be present if syncope was clearly correlated with coughing, micturition, defecation, or swallowing. ${ }^{6}$ The following circumstances were considered to be possible predisposing factors when syncope occurred in their presence and was clearly correlated with them: staying in a hot overcrowded room; a large meal especially with alcohol consumption; prolonged standing; and following exercise.

\section{STATISTICAL ANALYSIS}

Data are presented as mean (SD). Comparison of proportions was done by Fisher's exact test. Comparison between continuous variables was made by the $t$ test where the distribution of values was normal and by the non-parametric Mann-Whitney U test in cases of asymmetrical distribution. A p value $<0.05$ was considered significant.

\section{Results}

The recruitment process is summarised in fig 1. Of a total of 175 patients undergoing ATP and head up tilt tests, 121 (69\%) had a positive response to one or both tests; the final diagnosis remained unestablished in the remaining 54 


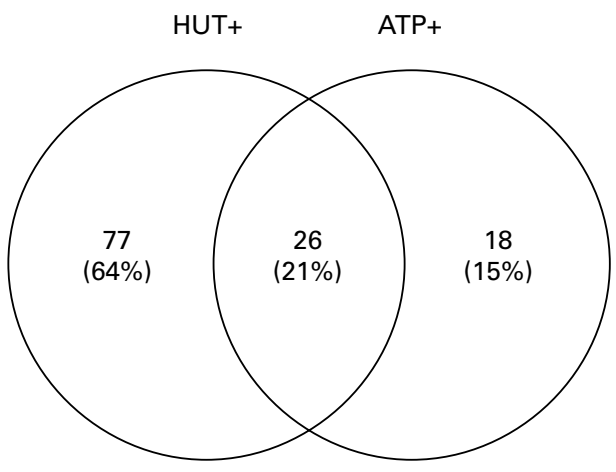

Figure 2 Distribution of the patients with a positive response to head up tilt or ATP or to both tests.

patients $(31 \%)$, who had a negative response to both tests. Head up tilt alone was positive in 77 patients, ATP alone was positive in 18 patients, and both tests were positive in 26 patients (fig 2). Thus a positive response to the head up tilt test was about four times more frequent than a positive response to the ATP test. An overlap was present in $21 \%$ of patients.

Owing to the different prevalence of the two forms of syncope, about one quarter of the patients with tilt induced syncope also had adenosine sensitive syncope, whereas more than half of the patients with adenosine sensitive syncope also had tilt induced syncope.

The clinical characteristics of these three groups of patients are compared in table 1). Compared with the patients with isolated positive head up tilt test, those with isolated positive ATP test were older and had a higher prevalence of associated diseases. However, when corrected for age, the presence of structural heart disease was no longer predictive of a positive response to ATP testing (MantelHaenszel $\chi^{2}$ test: $\left.p=0.77\right)$. Moreover, the patients with isolated positive ATP test had a very much shorter duration and fewer syncopal episodes, a lower prevalence of situational or vasovagal episodes, and a lower prevalence of triggering episodes; the onset of syncopal episodes was more often abrupt, without warning, and usually occurred in the standing position. The patients in whom both tests were positive had roughly intermediate characteristics. They differed from those with isolated positive head up tilt with regard to age, sex, and associated diseases, and had a lower prevalence of vasovagal, situational, and triggering factors. On the other hand, they also differed from those with isolated positive ATP test with regard to age, number and duration of syncopal episodes, and body position at the time of syncope occurrence; moreover, there was a trend toward a higher prevalence of vasovagal, situational, and triggering factors.

Of the 175 patients who underwent ATP and head up tilt testing, 61 had a history of vasovagal or situational episodes and 114 did not. Head up tilt was more often positive in those with a history of vasovagal or situational episodes $(67 \%$ v $54 \%, \mathrm{p}=0.07)$, whereas the ATP test was more often positive in patients without a history of vasovagal or situational episodes $(31 \% v 15 \%, \mathrm{p}=0.01)$.

THE MECHANISM OF POSITIVE TESTS

Atrioventricular block was present in 43 of 44 patients with a positive ATP test; only one patient had a positive response caused by a sinus arrest of $\geqslant 3 \mathrm{~s}$. A mixed or vasodepressor response was the most common response observed during the head up tilt test, occurring in 74 cases. Among the 29 cases with a cardioinhibitory response during head up tilt, sinus arrest of $\geqslant 3 \mathrm{~s}$ was more often observed than AV block $(82 \% \quad v \quad 18 \%$ of cases, respectively) (table 2). Among the patients with positive ATP test, a positive or negative response to head up tilt did not influence the type of response to ATP. Similarly, among the patients with positive head up tilt response, a positive or negative response to ATP did not influence the response to the head up tilt (table 2).

\section{Discussion}

The main conclusion of the study is that ATP and head up tilt tests identify different popula-

Table 1 Clinical characteristics of the three groups of patients with syncope

\begin{tabular}{|c|c|c|c|c|c|}
\hline & $\begin{array}{l}\text { Head up tilt }+ \\
(n=77)\end{array}$ & $\begin{array}{l}\text { Head up tilt }+ \\
\text { ATP+ }(n=26)\end{array}$ & $p$ Value $^{*}$ & $A T P+(n=18)$ & $p$ Value $\dagger$ \\
\hline Age (years) (mean (SD)) & $45(20)$ & $58(18)$ & 0.004 & $68(10)$ & $0.000 \ddagger$ \\
\hline Women $(\%)$ & $39(51 \%)$ & $19(73 \%)$ & 0.03 & $11(61 \%)$ & NS \\
\hline \multicolumn{6}{|l|}{ Associated diseases } \\
\hline Systemic hypertension & $4(5 \%)$ & $4(15 \%)$ & NS & $4(22 \%)$ & 0.04 \\
\hline Structural heart diseases & $10(13 \%)$ & $8(31 \%)$ & 0.004 & $5(28 \%)$ & NS \\
\hline ECG abnormalities & $7(9 \%)$ & $5(19 \%)$ & NS & $5(28 \%)$ & 0.05 \\
\hline \multicolumn{6}{|l|}{ History of syncopal episodes } \\
\hline $\begin{array}{l}\text { Total number (median } \\
\quad \text { (interquartile range)) }\end{array}$ & $3(2$ to 5$)$ & $4(2$ to 5$)$ & NS & $2(1$ to 3$)$ & $0.04 \ddagger$ \\
\hline $\begin{array}{l}\text { Duration (months) (median } \\
\text { (interquartile range)) }\end{array}$ & $36(6$ to 120$)$ & 42 (6 to 72$)$ & NS & $4(1$ to 12$)$ & $0.003 \ddagger$ \\
\hline Situational symptoms & $14(18 \%)$ & $4(15 \%)$ & NS & $2(11 \%)$ & NS \\
\hline Vasovagal symptoms & $23(30 \%)$ & $5(19 \%)$ & NS & $0(0 \%)$ & 0.004 \\
\hline Triggering factors & $22(29 \%)$ & $2(8 \%)$ & 0.02 & $0(0 \%)$ & 0.005 \\
\hline$>1$ of the above findings & $49(64 \%)$ & $8(31 \%)$ & 0.004 & $2(11 \%)$ & 0.000 \\
\hline Warnings & $55(71 \%)$ & $18(69 \%)$ & NS & $8(44 \%)$ & 0.03 \\
\hline Presyncopal episodes & $29(38 \%)$ & $12(46 \%)$ & NS & $4(22 \%)$ & NS \\
\hline Secondary trauma & $32(42 \%)$ & $12(46 \%)$ & NS & $8(44 \%)$ & NS \\
\hline$>1$ episode supine/sitting & $19(25 \%)$ & $9(35 \%)$ & NS & $1(6 \%)$ & NS $(0.06) \ddagger$ \\
\hline$>1$ episode standing & $66(86 \%)$ & $22(85 \%)$ & NS & $17(94 \%)$ & NS \\
\hline
\end{tabular}

* Statistical comparison between head up tilt+ group and head up tilt+/ATP+ group. +Statistical comparison between head up tilt+ group and ATP+ group.

$\ddagger$ Statistical comparison between ATP + group and head up tilt $+/$ ATP + group: $p<0.05$ 
Table 2 The characteristics of the positive resposes of ATP and head up tilt tests in the three groups of patients

\begin{tabular}{|c|c|c|c|c|}
\hline & $\begin{array}{l}\text { Head up tilt+ } \\
(n=77)\end{array}$ & $\begin{array}{l}\text { Head up tilt }+ \\
\text { ATP+ }(n=26)\end{array}$ & $A T P+(n=18)$ & $p$ Value \\
\hline \multicolumn{5}{|l|}{ ATP testing } \\
\hline Atrioventricular block (3rd degree) & - & $25(96 \%)$ & $18(100 \%)$ & NS \\
\hline Sinus pause $\geqslant 3$ seconds & - & $1(6 \%)$ & $0(0 \%)$ & NS \\
\hline Maximum RR interval (s) & - & $8.2(2.0)$ & $7.8(2.6)$ & NS \\
\hline Systolic blood pressure drop ( $\mathrm{mmHg}$ ) & - & $59(22)$ & $57(25)$ & NS \\
\hline \multicolumn{5}{|l|}{ Head up tilt testing } \\
\hline Passive phase & $17(22 \%)$ & $8(31 \%)$ & & NS \\
\hline Glyceryl trinitrate & $60(78 \%)$ & $18(69 \%)$ & & NS \\
\hline Atrioventricular block (3rd degree) & $4(5 \%)$ & $2(8 \%)$ & - & NS \\
\hline Sinus pause $\geqslant 3$ seconds & $18(23 \%)$ & $5(19 \%)$ & _ & NS \\
\hline \multicolumn{5}{|l|}{ Maximum RR interval (seconds) (median } \\
\hline (interquartile range)) & $6.1(3.7$ to 8.0$)$ & $6.8(4$ to 17$)$ & - & NS \\
\hline Mixed or vasodepressor type & $55(71 \%)$ & $19(73 \%)$ & - & NS \\
\hline
\end{tabular}

Values are mean (SD) or $\mathrm{n}(\%)$ unless stated.

tions of patients affected by syncope; thus different general clinical features, different histories of the syncopal episodes, and different sites of action on cardiac effectors were observed. Therefore adenosine sensitive syncope and tilt induced vasovagal syncope are two distinct clinical entities, probably with different aetiologies. Nevertheless, there is an important overlap between the two syndromes which makes it likely that there are some common physiopathological pathways.

\section{CLINICAL FEATURES OF ADENOSINE SENSITIVE} SYNCOPE

This is an uncommon form of syncope, four times less frequent than tilt induced vasovagal syncope. Indeed, in the present study it accounted for only $3 \%$ of patients referred for investigation of syncope and for $24 \%$ of the patients with a negative work up including head up tilt. These figures are similar to the $3.4 \%$ and $28 \%$ rates, respectively, observed in our previous study. ${ }^{1}$ Adenosine sensitive syncope first manifests itself in old age, though it occasionally occurs in younger patients too. ${ }^{1} \mathrm{By}$ contrast, tilt induced vasovagal syncope can occur at any age; typically it begins in the teenage years and there may be a long period of life without recurrences. ${ }^{15}$ This explains why, in the present study, we found a great difference in symptom duration and in total number of syncopal episodes between the two groups of patients. There is a female predominance in adenosine sensitive syncope, ${ }^{14}$ but the reason for this is unclear. As the attacks nearly always occur in the standing position and warning symptoms are frequently absent, loss of consciousness often results in falls that cause injury. The lack of historical findings of vasovagal or situational episodes and the absence of triggering factors (as defined in Methods) characterise this form and clearly differentiate it from vasovagal syncope. However, advanced age, female predominance, sudden onset, and frequency of trauma also differentiate adenosine sensitive syncope from truly unexplained syncope (ATP and head up tilt negative). ${ }^{1}$ The clinical presentation and ECG manifestation of adenosine sensitive syncope mimic Stokes-Adams syncope complicating AV block. Indeed, the ATP was able to reproduce a spontaneous episode of paroxysmal AV block in patients who had a fortuitous
ECG recording of a syncopal episode and in whom all the conventional investigations (including electrophysiological study) were unremarkable. ${ }^{1}$ In both induced and spontaneous AV blocks, the onset of block was abrupt and was not preceded by other rhythm disturbances or sinus bradyarrhythmias. By contrast, when a spontaneous cardioinhibitory neurally mediated syncope was recorded, the pause was caused by either sinus arrest or AV block (as during head up tilt) and it was usually preceded by other bradyarrhythmias. ${ }^{8}$

Other than in patients affected by adenosine sensitive syncope alone, the ATP test is expected to be frequently positive in patients with vasovagal syncope, and ATP has been shown to be capable of triggering a vasovagal reaction in susceptible patients. ${ }^{2}{ }^{3}$ In our present study, about $25 \%$ of the patients with tilt induced syncope also had adenosine sensitive syncope, and the ATP test was positive in $15 \%$ of the patients with a history of syncope suggestive of a neurally mediated mechanism. The clinical features of the patients who had both positive head up tilt and positive ATP tests differed from those of the patients with tilt induced syncope alone and from those with adenosine sensitive syncope alone (table 2); thus these patients had clinical features that were atypical of both the vasovagal syndrome and adenosine induced syncope. This suggests that in this particular population more complex, multiple mechanisms are responsible for syncope and that syncopal attacks can be caused either by a vasovagal mechanism or by an adenosine mediated mechanism, or both.

MECHANISMS OF ATP AND HEAD UP TILT TESTS ATP and adenosine are released from myocardial cells under physiological and pathological conditions (for example in the case of myocardial oxygen supply-demand imbalance) and have similar effects. The negative chronotropic and dromotropic action of ATP is caused by its rapid catabolism to adenosine and the subsequent action of adenosine at purinoceptor sites. ${ }^{11} 1216$

In this study the cardiac effects of the ATP and head up tilt tests were quite different and independent of one another, the atrioventricular node being more susceptible to ATP and the sinus node more susceptible to head up tilt (table 2). This is in agreement with the view 
that the sites of action are different: membrane purinoceptors for ATP and muscarinic (acetylcholine) receptors for the vagal outflow induced by head up tilt. ${ }^{11}{ }^{12}$ Nevertheless, the fact that a positive response to both head up tilt and ATP was found in $21 \%$ of our cases suggests that some common physiopathological mechanism is present (table 2). Indeed, although the receptors are different, the cardiac actions of adenosine are remarkably similar to those of the neurotransmitter acetylcholine. Both acetylcholine and adenosine produce the same effects and share similar receptor-effector coupling systems, resulting in the activation of a specific outward potassium current $\left(\mathrm{IK}_{\mathrm{Ach}, \mathrm{Ado}}\right)$ from the target effector cells. Moreover, a major role of acetylcholine and adenosine, in addition to their direct effect, is to function in parallel to oppose the cardiac stimulatory action of the sympathetic neurotransmitters noradrenaline (norepinephrine) and adrenaline (epinephrine) on adenyl cyclase (cAMP dependent effect). ${ }^{11}{ }^{12}$ Thus adrenergic, cholinergic, and purinergic outflows are integrated at the level of the receptor-effector coupling system, and the final cardiac effect results from the sum of these excitatory and inhibitory effects. A practical consequence might be that vasovagal syncope could be facilitated by an increased susceptibility to adenosine, and that adenosine sensitive syncope could be facilitated by an increased vagal outflow.

The cause of the hypersensitivity to exogenous adenosine found in the patients with adenosine sensitive syncope is not clear, as we carefully excluded all those patients with manifest or subtle AV conduction disorders or who were taking drugs that block AV conduction. There are several potential explanations for the observed increase in susceptibility to adenosine: increased density of A1 adenosine receptors in the AV node; increased coupling efficacy of the receptors; increased density of $\mathrm{IK}_{\mathrm{ado}}$; increased release of adenosine (that is, increased interstitial levels of adenosine); decreased degradation of adenosine; and the presence of constitutively active A1 receptors in the $\mathrm{AV}$ node. ${ }^{12}$ Nevertheless, we cannot exclude the possibility that unknown AV conduction abnormalities not recognisable by means of standard clinical and electrophysiological evaluation, or a non-specific susceptibility of the AV node to different triggers (for example vagal hyperactivity, as discussed above), could account for hypersensitivity to adenosine.

1 Brignole M, Gaggioli G, Menozzi C, et al. Adenosineinduced atrioventricular block in patients with unexplained syncope. The diagnostic value of ATP testing. Circulation 1997;96:3921-7.

2 Shen WK, Hammill S, Munger T, et al. Adenosine:potential modulator for vasovagal syncope. $\mathcal{F} \mathrm{Am}$ Coll Cardiol 1996;28:146-54

3 Mittal S, Stein K, Markowitz S, et al. Induction of neurally mediated syncope with adenosine. Circulation 1999;99: 1318-24.

4 Flammang D, Church T, Waynberger M, et al. Can adenosine 5 ' triphosphate be used to select treatment in severe vasovagal syndrome? Circulation 1997;96:1201-8.

5 Krol R, Morady F, Flaker G, et al. Electrophysiologic testing in patients with unexplained syncope: clinical and noninvasive predictors of outcome. $\mathcal{F}$ Am Coll Cardiol 1987;10: 358-63.

6 Kapoor W, Karpf M, Wieand S, et al. A prospective evaluation and follow-up of patients with syncope. $N$ Engl f Med 1983;309:197-204.

7 Brignole M, Menozzi C, Gianfranchi L, et al. Carotid sinus massage, eye-ball compression test and head up tilt test in patients with syncope of uncertain origin and in healthy patients with syncope of uncertain origin and in

8 Brignole M, Menozzi C, Bottoni N, et al. Mechanisms of syncope caused by transient bradycardia and the diagnostic value of electrophysiologic testing and cardiovascular reflexivity maneuvers. Am $\mathcal{F}$ Cardiol 1995;76:273-8.

9 Friedman DB, Jensen FB, Matzen S, et al. Non-invasive blood pressure monitoring during head up tilt test using the Penaz principle. Acta Anesthesiol Scand 1990;34:51922.

10 Petersen MEV, Williams TR, Sutton R. A comparison of non-invasive continuous finger blood pressure measurements (Finapres) with intra-arterial pressure during prolonged head up tilt. Eur Heart f 1995;16:1647-54.

11 Lerman B, Belardinelli L. Cardiac electrophysiology of adenosine. Basic and clinical concepts. Circulation 1991;83: 1499-508.

12 Belardinelli L, Linden J, Berne RM. The cardiac effects of adenosine. Prog Cardiovasc Dis 1989;22:73-97.

13 Favale S, Di Biase M, Rizzo U, et al. Effect of adenosine and adenosine 5'-triphosphate on atrioventricular conduction in patients. F Am Coll Cardiol 1985;5:1212-19.

14 Raviele A, Menozzi C, Brignole M, et al. Value of head up tilt testing potentiated with sublingual nitroglycerin to assess the origin of unexplained syncope. Am $\mathcal{F}$ Cardiol 1995;76:267-72.

15 Sutton R. Vasovagal syncope:clinical features, epidemiology, and natural history. In: Blanc JJ, Benditt D, Sutton R, eds. Neurally mediated syncope: pathophysiology, inves
treatment. Armonk (NY): Futura, 1996:71-6.

16 Pelleg A, Mitsuoka T, Michelson E, et al. Adenosine mediates the negative chronotropic action of adenosine 5 '-triphosphate in the canine sinus node. 7 Pharmacol Exp Ther 1987;242:791-5. 